\title{
Steering laws for motion camouflage
}

\author{
E. W. Justh \\ Institute for Systems Research \\ University of Maryland \\ College Park, MD 20742, USA \\ justh@umd.edu
}

\author{
P. S. Krishnaprasad \\ Institute for Systems Research and \\ Dept. of Electrical and Computer Engineering \\ University of Maryland \\ College Park, MD 20742, USA \\ krishna@umd.edu
}

\begin{abstract}
Motion camouflage is a stealth strategy observed in nature. We formulate the problem as a feedback system for particles moving at constant speed, and define what it means for the system to be in a state of motion camouflage. (Here we focus on the planar setting, although the results can be generalized to three-dimensional motion.) We propose a biologically plausible feedback law, and use a high-gain limit to prove accessibility of a motion camouflage state in finite time. We discuss connections to work in missile guidance. We also present simulation results to explore the performance of the motion camouflage feedback law for a variety of settings.
\end{abstract}

\section{INTRODUCTION}

Motion camouflage is a stealth strategy employed by various visual insects and animals to achieve prey capture, mating or territorial combat. In one type of motion camouflage, the predator camouflages itself against a fixed background object so that the prey observes no relative motion between the predator and the fixed object. In the other type of motion camouflage, the predator approaches the prey such that from the point of view of the prey, the predator always appears to be at the same bearing. (In this case, we say that the object against which the predator is camouflaged is the point at infinity.) Assuming that the prey can readily observe optical flow, but only poorly sense looming, this type of motion by the predator is then difficult to detect by the prey. For example, insects with compound eyes are quite sensitive to optical flow (which arises from the transverse component of the relative velocity between the predator and the prey), but are far less sensitive to slight changes in the size of images (which arise from the component of the relative velocity between the predator and prey along the line between them). More broadly such interactions may also apply in settings of mating activity or territorial maneuvers as well. In the work, [1] of Srinivasan and Davey, it was suggested that the data on visually mediated interactions between two hoverflies, Syritta pipiens obtained earlier by Collett and Land [2], supports a motion camouflage hypothesis. Later, Mizutani, Chahl and Srinivasan [3], observing territorial aerial maneuvers of dragonflies Hemianax papuensis, concluded that the flight pattern is motivated by motion camouflage (see Figure 1 in their paper). See also [4] for a review of related themes in insect vision and flight control.

Motion camouflage can be used by a predator to stealthily pursue prey, but a motion camouflage strategy can also be used by the prey to evade a predator. The only difference between the strategy of the predator and the strategy of the evader is that the predator seeks to approach the prey while maintaining motion camouflage, whereas the evader seeks to move away from the predator while maintaining motion camouflage. Besides explaining certain biological pursuit strategies, motion camouflage may also be quite useful in certain military scenarios (although the "predator" and "prey" labels may not be descriptive). In some settings, as is the case in [1], [2], [3] it is more appropriate to substitute the labels "shadower" and "shadowee" for the predator-prey terminology.

In this work, we take a structured approach to deriving feedback laws for motion camouflage, which incorporate biologically plausible (vision) sensor measurements. We model the predator and prey as point particles moving at constant (but different) speeds, and subject to steering (curvature) control. For an appropriate choice of feedback control law for one of the particles (as the other follows a prescribed trajectory), a state of motion camouflage is then approached as the system evolves. (In the situation where the predator follows a motioncamouflage law, and the speed of the predator exceeds the speed of the prey, the predator is able to pass "close" to the prey in finite time. In practice, once the predator is sufficiently close to the prey, it would change its strategy from a pursuit strategy to an intercept strategy.)

What distinguishes this work from earlier study of motioncamouflage trajectories in [5] is that we present biologically plausible feedback laws leading to motion camouflage. Furthermore, unlike the neural-network approach used in [6] to achieve motion camouflage using biologically-plausible sensor data, our approach gives an explicit form for the feedback law which has a straightforward physical interpretation.

The study of motion camouflage problems also naturally extends earlier work on interacting systems of particles, using the language of curves and moving frames [7]-[11].

\section{PLANAR PURSUIT-EVASION MODEL}

For concreteness, we consider the problem of motion camouflage in which the predator (which we refer to as the "pursuer") attempts to intercept the prey (which we refer to as the "evader") while appearing to the prey as though it is always at the same bearing (i.e., motion camouflaged against the point at infinity). In the model we consider, the pursuer moves at unit speed in the plane, while the evader moves at a constant speed $\nu<1$. The dynamics of the pursuer are given 


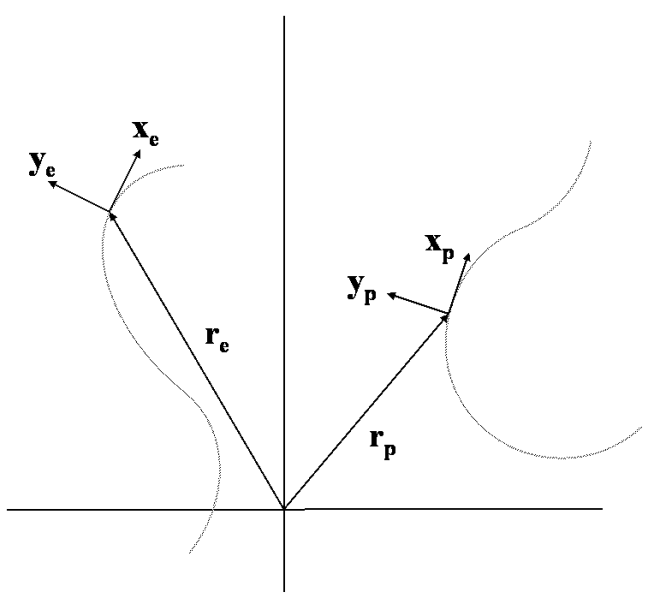

Fig. 1. Planar trajectories for the pursuer and evader, and their respective natural Frenet frames.

by

$$
\begin{aligned}
\dot{\mathbf{r}}_{p} & =\mathbf{x}_{p}, \\
\dot{\mathbf{x}}_{p} & =\mathbf{y}_{p} u_{p}, \\
\dot{\mathbf{y}}_{p} & =-\mathbf{x}_{p} u_{p},
\end{aligned}
$$

where $\mathbf{r}_{p}$ is the position of the pursuer, $\mathbf{x}_{p}$ is the unit tangent vector to the trajectory of the pursuer, $\mathbf{y}_{p}$ is the corresponding unit normal vector (which completes a right-handed orthonormal basis with $\mathbf{x}_{p}$ ), and the plane curvature $u_{p}$ is the steering control for the pursuer. Similarly, the dynamics of the evader are

$$
\begin{aligned}
\dot{\mathbf{r}}_{e} & =\nu \mathbf{x}_{e}, \\
\dot{\mathbf{x}}_{e} & =\nu \mathbf{y}_{e} u_{e}, \\
\dot{\mathbf{y}}_{e} & =-\nu \mathbf{x}_{e} u_{e},
\end{aligned}
$$

where $\mathbf{r}_{e}$ is the position of the evader, $\mathbf{x}_{e}$ is the unit tangent vector to the trajectory of the evader, $\mathbf{y}_{e}$ is the corresponding unit normal vector, and $u_{e}$ is the steering control for the evader. Figure 1 illustrates equations (1) and (2). Note that $\left\{\mathbf{x}_{p}, \mathbf{y}_{p}\right\}$ and $\left\{\mathbf{x}_{e}, \mathbf{y}_{e}\right\}$ are planar natural Frenet frames for the trajectories of the pursuer and evader, respectively.

We model the pursuer and evader as point particles (confined to the plane), and use natural frames and curvature controls to describe their motion, because this is a simple model for which we can derive both physical intuition and concrete control laws. (Furthermore, although we save the details for a future paper, this approach generalizes nicely for three-dimensional motion.) Flying insects and animals (also unmanned aerial vehicles) have limited maneuverability and must maintain sufficient airspeed to stay aloft, so treating their motion as constant-speed with steering control is physically reasonable, at least for some range of flight conditions. (Note that the steering control directly drives the angular velocity of the particle, and hence is actually an acceleration input. However, this acceleration is constrained to be perpendicular to the instantaneous direction of motion, and therefore the speed remains unchanged.)
We refer to (1) and (2) as the "pursuit-evader system." In what follows, we assume that the pursuer follows a feedback strategy to drive the system toward a state of motion camouflage, and close in on the evader. The evader, on the other hand, follows an open-loop strategy. The analysis we present for the pursuer feedback strategy also suggests (with a sign change in the control law) how the evader could use feedback and a motion-camouflage strategy to conceal its flight from the pursuer. Ultimately, it would be interesting to address the game-theoretic problem in which both the pursuer and evader follow feedback strategies, so that the system would truly be a pursuit-evader system. (What we address in this work would be more properly described as a pursuer-pursuee system. However, we keep the pursuer-evader terminology, because it sets the stage for analyzing the true pursuer-evader system, which we plan to address in a future paper.)

\section{A. Motion camouflage with respect to the point at infinity}

Motion camouflage with respect to the point at infinity is given by

$$
\mathbf{r}_{p}=\mathbf{r}_{e}+\lambda \mathbf{r}_{\infty}
$$

where $\mathbf{r}_{\infty}$ is a fixed unit vector and $\lambda$ is a time-dependent scalar (see also Section 5 of [5]).

Let

$$
\mathbf{r}=\mathbf{r}_{p}-\mathbf{r}_{e}
$$

be the vector from the evader to the pursuer. We refer to $\mathbf{r}$ as the "baseline vector," and $|\mathbf{r}|$ as the "baseline length." We restrict attention to non-collision states, i.e., $\mathbf{r} \neq 0$. In that case, the component of the pursuer velocity $\dot{\mathbf{r}}_{p}$ transverse to the base line is

$$
\dot{\mathbf{r}}_{p}-\left(\frac{\mathbf{r}}{|\mathbf{r}|} \cdot \dot{\mathbf{r}}_{p}\right) \frac{\mathbf{r}}{|\mathbf{r}|},
$$

and similarly, that of the evader is

$$
\dot{\mathbf{r}}_{e}-\left(\frac{\mathbf{r}}{|\mathbf{r}|} \cdot \dot{\mathbf{r}}_{e}\right) \frac{\mathbf{r}}{|\mathbf{r}|}
$$

The relative transverse component is

$$
\begin{aligned}
\mathbf{w} & =\left(\dot{\mathbf{r}}_{p}-\dot{\mathbf{r}}_{e}\right)-\left(\frac{\mathbf{r}}{|\mathbf{r}|} \cdot\left(\dot{\mathbf{r}}_{p}-\dot{\mathbf{r}}_{e}\right)\right) \frac{\mathbf{r}}{|\mathbf{r}|} \\
& =\dot{\mathbf{r}}-\left(\frac{\mathbf{r}}{|\mathbf{r}|} \cdot \dot{\mathbf{r}}\right) \frac{\mathbf{r}}{|\mathbf{r}|} .
\end{aligned}
$$

Lemma (Infinitesimal characterization of motion camouflage): The pursuit-evasion system (1), 2) is in a state of motion camouflage without collision on an interval iff $\mathbf{w}=0$ on that interval.

Proof: $(\Longrightarrow)$ Suppose motion camouflage holds. Thus

$$
\mathbf{r}(t)=\lambda(t) \mathbf{r}_{\infty}, \quad t \in[0, T] .
$$

Differentiating, $\dot{\mathbf{r}}=\dot{\lambda} \mathbf{r}_{\infty}$. Hence,

$$
\begin{aligned}
\mathbf{w} & =\dot{\mathbf{r}}-\left(\frac{\mathbf{r}}{|\mathbf{r}|} \cdot \dot{\mathbf{r}}\right) \frac{\mathbf{r}}{|\mathbf{r}|} \\
& =\dot{\lambda} \mathbf{r}_{\infty}-\left(\frac{\lambda}{|\lambda|} \mathbf{r}_{\infty} \cdot \dot{\lambda} \mathbf{r}_{\infty}\right) \frac{\lambda}{|\lambda|} \mathbf{r}_{\infty} \\
& =0 \text { on }[0, T] .
\end{aligned}
$$


$(\Longleftarrow)$ Suppose $\mathbf{w}=0$ on $[0, T]$. Thus

$$
\dot{\mathbf{r}}=\left(\frac{\mathbf{r}}{|\mathbf{r}|} \cdot \dot{\mathbf{r}}\right) \frac{\mathbf{r}}{|\mathbf{r}|} \triangleq \xi \mathbf{r},
$$

so that

$$
\begin{aligned}
\mathbf{r}(t) & =\exp \left(\int_{0}^{t} \xi(\sigma) d \sigma\right) \mathbf{r}(0) \\
& =|\mathbf{r}(0)| \exp \left(\int_{0}^{t} \xi(\sigma) d \sigma\right) \frac{\mathbf{r}(0)}{|\mathbf{r}(0)|} \\
& =\lambda(t) \mathbf{r}_{\infty},
\end{aligned}
$$

where $\mathbf{r}_{\infty}=\mathbf{r}(0) /|\mathbf{r}(0)|$ and $\lambda(t)=|\mathbf{r}(0)| \exp \left(\int_{0}^{t} \xi(\sigma) d \sigma\right)$.

It follows from the Lemma that the set of all motion camouflage states constitutes a 5-dimensional smooth manifold with two connected components, each diffeomorphic to $S^{1} \times \mathbb{R} \times$ $S E(2)$ in the 6-dimensional state space $S E(2) \times S E(2)$ of the problem. In practice we are interested in how far the pursuitevasion system is from a state of motion camouflage. In what follows, we offer a measure of this.

\section{B. Cost function}

Consider the ratio

$$
\Gamma(t)=\frac{\frac{d}{d t}|\mathbf{r}|}{\left|\frac{d \mathbf{r}}{d t}\right|}
$$

which compares the rate of change of the baseline length to the absolute rate of change of the baseline vector. If the baseline experiences pure lengthening, then the ratio assumes its maximum value, $\Gamma(t)=1$. If the baseline experiences pure shortening, then the ratio assumes its minimum value, $\Gamma(t)=-1$. If the baseline experiences pure rotation, but remains the same length, then $\Gamma(t)=0$. Noting that

$$
\frac{d}{d t}|\mathbf{r}|=\frac{\mathbf{r}}{|\mathbf{r}|} \cdot \dot{\mathbf{r}}
$$

we see that $\Gamma(t)$ may alternatively be written as

$$
\Gamma(t)=\frac{\mathbf{r}}{|\mathbf{r}|} \cdot \frac{\dot{\mathbf{r}}}{|\dot{\mathbf{r}}|} .
$$

Thus, $\Gamma(t)$ is the dot product of two unit vectors: one in the direction of $\mathbf{r}$, and the other in the direction of $\dot{\mathbf{r}}$. Note that $\Gamma$ is well-defined except at $\mathbf{r}=0$, since

$$
1-\nu \leq|\dot{\mathbf{r}}|=\left|\mathbf{x}_{p}-\nu \mathbf{x}_{e}\right| \leq 1+\nu .
$$

For convenience, we define the notation $\mathbf{q}^{\perp}$ to represent the vector $\mathbf{q}$ rotated counter-clockwise in the plane by an angle $\pi / 2$. Thus, for example, $\mathbf{x}_{p}^{\perp}=\mathbf{y}_{p}$. The transverse component $\mathbf{w}$ of relative velocity, expression (5), then becomes

$$
\begin{aligned}
\mathbf{w} & =\dot{\mathbf{r}}-\left(\frac{\mathbf{r}}{|\mathbf{r}|} \cdot \dot{\mathbf{r}}\right) \frac{\mathbf{r}}{|\mathbf{r}|}=\left[\left(\frac{\mathbf{r}}{|\mathbf{r}|}\right)^{\perp} \cdot \dot{\mathbf{r}}\right]\left(\frac{\mathbf{r}}{|\mathbf{r}|}\right)^{\perp} \\
& =-\left(\frac{\mathbf{r}}{|\mathbf{r}|} \cdot \dot{\mathbf{r}}^{\perp}\right)\left(\frac{\mathbf{r}}{|\mathbf{r}|}\right)^{\perp} .
\end{aligned}
$$

For convenience, we define $w$ to be the (signed) magnitude of w, i.e.,

$$
w=\mathbf{w} \cdot\left(\frac{\mathbf{r}}{|\mathbf{r}|}\right)^{\perp}=-\left(\frac{\mathbf{r}}{|\mathbf{r}|} \cdot \dot{\mathbf{r}}^{\perp}\right),
$$

and refer also to $w$ as the transverse component of the relative velocity. From the orthogonal decomposition

$$
\frac{\mathbf{r}}{|\mathbf{r}|}=\left(\frac{\mathbf{r}}{|\mathbf{r}|} \cdot \frac{\dot{\mathbf{r}}}{|\dot{\mathbf{r}}|}\right)\left(\frac{\dot{\mathbf{r}}}{|\dot{\mathbf{r}}|}\right)+\left[\frac{\mathbf{r}}{|\mathbf{r}|} \cdot\left(\frac{\dot{\mathbf{r}}}{|\dot{\mathbf{r}}|}\right)^{\perp}\right]\left(\frac{\dot{\mathbf{r}}}{|\dot{\mathbf{r}}|}\right)^{\perp},
$$

it follows that

$$
1=\left(\frac{\mathbf{r}}{|\mathbf{r}|} \cdot \frac{\dot{\mathbf{r}}}{|\dot{\mathbf{r}}|}\right)^{2}+\left[\frac{\mathbf{r}}{|\mathbf{r}|} \cdot\left(\frac{\dot{\mathbf{r}}}{|\dot{\mathbf{r}}|}\right)^{\perp}\right]^{2}=\Gamma^{2}+\frac{|w|^{2}}{|\dot{\mathbf{r}}|^{2}} .
$$

Thus $\left(1-\Gamma^{2}\right)$ is a measure of the distance from motion camouflage.

\section{Feedback law derivation}

Differentiating $\Gamma$ along trajectories of (1) and (2) gives

$$
\begin{aligned}
\dot{\Gamma}= & \left(\frac{\dot{\mathbf{r}} \cdot \dot{\mathbf{r}}+\mathbf{r} \cdot \ddot{\mathbf{r}}}{|\mathbf{r}||\dot{\mathbf{r}}|}\right)-\left(\frac{\mathbf{r} \cdot \dot{\mathbf{r}}}{|\dot{\mathbf{r}}|}\right)\left(\frac{\mathbf{r} \cdot \dot{\mathbf{r}}}{|\mathbf{r}|^{3}}\right)-\left(\frac{\mathbf{r} \cdot \dot{\mathbf{r}}}{|\mathbf{r}|}\right)\left(\frac{\dot{\mathbf{r}} \cdot \ddot{\mathbf{r}}}{|\dot{\mathbf{r}}|^{3}}\right) \\
= & \frac{|\dot{\mathbf{r}}|}{|\mathbf{r}|}\left[1-\left(\frac{\mathbf{r}}{|\mathbf{r}|} \cdot \frac{\dot{\mathbf{r}}}{|\dot{\mathbf{r}}|}\right)^{2}\right] \\
& +\frac{1}{|\dot{\mathbf{r}}|}\left[\frac{\mathbf{r}}{|\mathbf{r}|}-\left(\frac{\mathbf{r}}{|\mathbf{r}|} \cdot \frac{\dot{\mathbf{r}}}{|\dot{\mathbf{r}}|}\right) \frac{\dot{\mathbf{r}}}{|\dot{\mathbf{r}}|}\right] \cdot \ddot{\mathbf{r}}
\end{aligned}
$$

From (4) we obtain

$$
\dot{\mathbf{r}}^{\perp}=\mathbf{y}_{p}-\nu \mathbf{y}_{e},
$$

and

$$
\ddot{\mathbf{r}}=\mathbf{y}_{p} u_{p}-\nu^{2} \mathbf{y}_{e} u_{e} .
$$

Also,

$$
\begin{aligned}
{\left[\frac{\mathbf{r}}{|\mathbf{r}|}-\left(\frac{\mathbf{r}}{|\mathbf{r}|} \cdot \frac{\dot{\mathbf{r}}}{|\dot{\mathbf{r}}|}\right) \frac{\dot{\mathbf{r}}}{|\dot{\mathbf{r}}|}\right] } & =\left[\frac{\mathbf{r}}{|\mathbf{r}|} \cdot\left(\frac{\dot{\mathbf{r}}}{|\dot{\mathbf{r}}|}\right)^{\perp}\right]\left(\frac{\dot{\mathbf{r}}}{|\dot{\mathbf{r}}|}\right)^{\perp} \\
& =\frac{1}{|\dot{\mathbf{r}}|^{2}}\left(\frac{\mathbf{r}}{|\mathbf{r}|} \cdot \dot{\mathbf{r}}^{\perp}\right) \dot{\mathbf{r}}^{\perp}
\end{aligned}
$$

Then from (18) we obtain

$$
\begin{aligned}
\dot{\Gamma}= & \frac{|\dot{\mathbf{r}}|}{|\mathbf{r}|}\left[1-\left(\frac{\mathbf{r}}{|\mathbf{r}|} \cdot \frac{\dot{\mathbf{r}}}{|\dot{\mathbf{r}}|}\right)^{2}\right] \\
+ & \frac{1}{|\dot{\mathbf{r}}|}\left[\frac{\mathbf{r}}{|\mathbf{r}|}-\left(\frac{\mathbf{r}}{|\mathbf{r}|} \cdot \frac{\dot{\mathbf{r}}}{|\dot{\mathbf{r}}|}\right) \frac{\dot{\mathbf{r}}}{|\dot{\mathbf{r}}|}\right] \cdot\left(\mathbf{y}_{p} u_{p}-\nu^{2} \mathbf{y}_{e} u_{e}\right) \\
= & \frac{|\dot{\mathbf{r}}|}{|\mathbf{r}|}\left[\frac{1}{|\dot{\mathbf{r}}|^{2}}\left(\frac{\mathbf{r}}{|\mathbf{r}|} \cdot \dot{\mathbf{r}}^{\perp}\right)^{2}\right] \\
+ & \frac{1}{|\dot{\mathbf{r}}|}\left[\frac{1}{|\dot{\mathbf{r}}|^{2}}\left(\frac{\mathbf{r}}{|\mathbf{r}|} \cdot \dot{\mathbf{r}}^{\perp}\right) \dot{\mathbf{r}}^{\perp}\right] \cdot\left(\mathbf{y}_{p} u_{p}-\nu^{2} \mathbf{y}_{e} u_{e}\right) .
\end{aligned}
$$

Noting that

$$
\dot{\mathbf{r}}^{\perp} \cdot \mathbf{y}_{p}=\dot{\mathbf{r}} \cdot \mathbf{x}_{p}=1-\nu\left(\mathbf{x}_{p} \cdot \mathbf{x}_{e}\right) \geq 1-\nu>0,
$$

and

$$
\dot{\mathbf{r}}^{\perp} \cdot \mathbf{y}_{e}=\dot{\mathbf{r}} \cdot \mathbf{x}_{e}=\left(\mathbf{x}_{p} \cdot \mathbf{x}_{e}\right)-\nu
$$


we obtain

$$
\begin{aligned}
\dot{\Gamma}=\frac{|\dot{\mathbf{r}}|}{|\mathbf{r}|}\left[\frac{1}{|\dot{\mathbf{r}}|^{2}}\left(\frac{\mathbf{r}}{|\mathbf{r}|} \cdot \dot{\mathbf{r}}^{\perp}\right)^{2}\right] \\
+\frac{1}{|\dot{\mathbf{r}}|}\left[\frac{1}{|\dot{\mathbf{r}}|^{2}}\left(\frac{\mathbf{r}}{|\mathbf{r}|} \cdot \dot{\mathbf{r}}^{\perp}\right)\right]\left(1-\nu\left(\mathbf{x}_{p} \cdot \mathbf{x}_{e}\right)\right) u_{p} \\
+\frac{1}{|\dot{\mathbf{r}}|}\left[\frac{1}{|\dot{\mathbf{r}}|^{2}}\left(\frac{\mathbf{r}}{|\mathbf{r}|} \cdot \dot{\mathbf{r}}^{\perp}\right)\right]\left(\nu-\left(\mathbf{x}_{p} \cdot \mathbf{x}_{e}\right)\right) \nu^{2} u_{e}
\end{aligned}
$$

Suppose that we take

$$
u_{p}=-\mu\left(\frac{\mathbf{r}}{|\mathbf{r}|} \cdot \dot{\mathbf{r}}^{\perp}\right)+\left[\frac{\left(\mathbf{x}_{p} \cdot \mathbf{x}_{e}\right)-\nu}{1-\nu\left(\mathbf{x}_{p} \cdot \mathbf{x}_{e}\right)}\right] \nu^{2} u_{e},
$$

where $\mu>0$, so that the steering control for the pursuer consists of two terms: one involving the motion of the evader, and one involving the transverse component of the relative velocity. Then

$$
\dot{\Gamma}=-\left[\frac{\mu}{|\dot{\mathbf{r}}|}\left(1-\nu\left(\mathbf{x}_{p} \cdot \mathbf{x}_{e}\right)\right)-\frac{|\dot{\mathbf{r}}|}{|\mathbf{r}|}\right]\left[\frac{1}{|\dot{\mathbf{r}}|}\left(\frac{\mathbf{r}}{|\mathbf{r}|} \cdot \dot{\mathbf{r}}^{\perp}\right)\right]^{2},
$$

and for any choice of $\mu>0$, there exists $r_{o}>0$ such that

$$
\frac{\mu}{|\dot{\mathbf{r}}|}\left(1-\nu\left(\mathbf{x}_{p} \cdot \mathbf{x}_{e}\right)\right)-\frac{|\dot{\mathbf{r}}|}{|\mathbf{r}|}>0,
$$

for all $\mathbf{r}$ such that $|\mathbf{r}|>r_{o}$. Thus, for control law (26,

$$
\dot{\Gamma} \leq 0, \quad \forall|\mathbf{r}|>r_{o}
$$

\section{THE HIGH-GAIN LIMIT}

Control law (26) has the nice property that for any value of the gain $\mu>0$, there is a disc of radius $r_{o}$ (depending on $\mu$ ) such that $\dot{\Gamma} \leq 0$ outside the disc. However, the problem with (26) is that the pursuer needs to know (i.e., sense and estimate) the evader's steering program $u_{e}$. Here we show that by taking $\mu$ sufficiently large, motion camouflage can be achieved (in a sense we will make precise) using a control law depending only on the transverse relative velocity:

$$
u_{p}=-\mu\left(\frac{\mathbf{r}}{|\mathbf{r}|} \cdot \dot{\mathbf{r}}^{\perp}\right),
$$

in place of (26), provided $\left|u_{e}\right|$ is bounded. Comparing (30) to (15), we see that, indeed, $u_{p}$ is proportional to the signed length of the relative transverse velocity vector. We will designate this as the motion camouflage proportional guidance (MCPG) law for future reference (see Section V below).

As is further discussed in Section $V$, 30 requires range information as well as pure optical flow sensing. However, the range information can be coarse, since range errors (within appropriate bounds) have the same effect in (30) as gain variations. We say that (30) is biologically plausible because the only critical sensor measurement required is optical flow sensing. Optical flow sensing does not yield the relative transverse velocity directly, but rather the angular speed of the image of the evader across the pursuer's eye. In fact, it is the sign of the optical flow that is most critical to measure correctly, since errors in the magnitude of the optical flow, like range errors, only serve to modulate the gain in (30).
For biological systems, the capabilities of the sensors vis-a$v i s$ the sensing requirements for implementing (30) constrain the range of conditions for which 30 represents a feasible control strategy. In the high-gain limit we focus on below, sensor noise (which is amplified by the high gain) would be expected to have significant impact. However, to illustrate the essential behavior, here we neglect both sensor limitations and noise.

\section{A. Bounds and estimates}

Let us consider control law (30), and the resulting behavior of $\Gamma$ as a function of time. From (25), we obtain the inequality

$$
\begin{aligned}
\dot{\Gamma}=- & {\left[\frac{\mu}{|\dot{\mathbf{r}}|}\left(1-\nu\left(\mathbf{x}_{p} \cdot \mathbf{x}_{e}\right)\right)-\frac{|\dot{\mathbf{r}}|}{|\mathbf{r}|}\right]\left[\frac{1}{|\dot{\mathbf{r}}|}\left(\frac{\mathbf{r}}{|\mathbf{r}|} \cdot \dot{\mathbf{r}}^{\perp}\right)\right]^{2} } \\
& +\frac{1}{|\dot{\mathbf{r}}|}\left[\frac{1}{|\dot{\mathbf{r}}|^{2}}\left(\frac{\mathbf{r}}{|\mathbf{r}|} \cdot \dot{\mathbf{r}}^{\perp}\right)\right]\left(\nu-\left(\mathbf{x}_{p} \cdot \mathbf{x}_{e}\right)\right) \nu^{2} u_{e} \\
\leq- & \left(1-\Gamma^{2}\right)\left[\frac{\mu}{|\dot{\mathbf{r}}|}\left(1-\nu\left(\mathbf{x}_{p} \cdot \mathbf{x}_{e}\right)\right)-\frac{|\dot{\mathbf{r}}|}{|\mathbf{r}|}\right] \\
& +\frac{1}{|\dot{\mathbf{r}}|^{2}} \sqrt{1-\Gamma^{2}}\left|\left(\nu-\left(\mathbf{x}_{p} \cdot \mathbf{x}_{e}\right)\right) \nu^{2} u_{e}\right| \\
\leq- & \left(1-\Gamma^{2}\right)\left[\frac{\mu}{|\dot{\mathbf{r}}|}(1-\nu)-\frac{|\dot{\mathbf{r}}|}{|\mathbf{r}|}\right] \\
& +\left(\sqrt{1-\Gamma^{2}}\right) \frac{\nu^{2}(1+\nu)\left(\max \left|u_{e}\right|\right)}{|\dot{\mathbf{r}}|^{2}} \\
\leq- & \left(1-\Gamma^{2}\right)\left[\mu\left(\frac{1-\nu}{1+\nu}\right)-\frac{1+\nu}{|\mathbf{r}|}\right] \\
& +\left(\sqrt{1-\Gamma^{2}}\right)\left[\frac{\nu^{2}(1+\nu)\left(\max \left|u_{e}\right|\right)}{(1-\nu)^{2}}\right]
\end{aligned}
$$

where we have used (13). For convenience, we define the constant $c_{1}>0$ as

$$
c_{1}=\frac{\nu^{2}(1+\nu)\left(\max \left|u_{e}\right|\right)}{(1-\nu)^{2}} .
$$

For any $\mu>0$, we can define $r_{o}>0$ and $c_{o}>0$ such that

$$
\mu=\left(\frac{1+\nu}{1-\nu}\right)\left(\frac{1+\nu}{r_{o}}+c_{o}\right)
$$

(and it is clear that many such choices of $r_{o}$ and $c_{o}$ exist). Note that (33) implies

$$
\mu \geq\left(\frac{1+\nu}{1-\nu}\right)\left(\frac{1+\nu}{|\mathbf{r}|}+c_{o}\right), \quad \forall|\mathbf{r}| \geq r_{o} .
$$

Thus, for $|\mathbf{r}| \geq r_{o}$, 31 becomes

$$
\begin{aligned}
\dot{\Gamma} \leq & \left(1-\Gamma^{2}\right)\left[\left(\frac{1+\nu}{1-\nu}\right)\left(\frac{1+\nu}{|\mathbf{r}|}+c_{o}\right)\left(\frac{1-\nu}{1+\nu}\right)-\frac{1+\nu}{|\mathbf{r}|}\right] \\
& +\left(\sqrt{1-\Gamma^{2}}\right) c_{1} \\
= & -\left(1-\Gamma^{2}\right) c_{o}+\left(\sqrt{1-\Gamma^{2}}\right) c_{1} .
\end{aligned}
$$

Suppose that given $0<\epsilon<<1$, we take $c_{o} \geq 2 c_{1} / \sqrt{\epsilon}$. Then 
for $\left(1-\Gamma^{2}\right)>\epsilon$,

$$
\begin{aligned}
\dot{\Gamma} & \leq-\left(1-\Gamma^{2}\right) c_{o}+\left(\sqrt{1-\Gamma^{2}}\right) c_{1} \\
& =-\left(1-\Gamma^{2}\right)\left(c_{o}-\frac{c_{1}}{\sqrt{1-\Gamma^{2}}}\right) \\
& \leq-\left(1-\Gamma^{2}\right)\left(c_{o}-\frac{c_{1}}{\sqrt{\epsilon}}\right) \\
& =-\left(1-\Gamma^{2}\right) c_{2},
\end{aligned}
$$

where

$$
c_{2}=c_{o}-\frac{c_{1}}{\sqrt{\epsilon}}>0
$$

Remark: There are two possibilities for

$$
\left(1-\Gamma^{2}\right) \leq \epsilon
$$

The state we seek to drive the system toward has $\Gamma \approx-1$; however, 38 can also be satisfied for $\Gamma \approx 1$. (Recall that $-1 \leq \Gamma \leq 1$.) There is always a set of initial conditions such that $(38)$ is satisfied with $\Gamma \approx 1$. We can address this issue as follows: let $\epsilon_{o}>0$ denote how close to -1 we wish to drive $\Gamma$, and let $\Gamma_{0}=\Gamma(0)$ denote the initial value of $\Gamma$. Take

$$
\epsilon=\min \left(\epsilon_{o}, 1-\Gamma_{0}^{2}\right),
$$

so that 36 with 37 applies from time $t=0$.

From (36), we can write

$$
\frac{d \Gamma}{1-\Gamma^{2}} \leq-c_{2} d t
$$

which, integrating both sides, leads to

$$
\int_{\Gamma_{0}}^{\Gamma} \frac{d \tilde{\Gamma}}{1-\tilde{\Gamma}^{2}} \leq-c_{2} \int_{0}^{t} d \tilde{t}=-c_{2} t
$$

where $\Gamma_{0}=\Gamma(t=0)$. Noting that

$$
\int_{\Gamma_{0}}^{\Gamma} \frac{d \tilde{\Gamma}}{1-\tilde{\Gamma}^{2}}=\int_{\Gamma_{0}}^{\Gamma} d\left(\tanh ^{-1} \tilde{\Gamma}\right)=\tanh ^{-1} \Gamma-\tanh ^{-1} \Gamma_{0},
$$

we see that for $|\mathbf{r}| \geq r_{o}$, 36 implies

$$
\Gamma(t) \leq \tanh \left(\tanh ^{-1} \Gamma_{0}-c_{2} t\right),
$$

where we have used the fact that $\tanh ^{-1}(\cdot)$ is a monotone increasing function.

Now we consider estimating how long $|\mathbf{r}| \geq r_{o}$, which in turn determines how large $t$ can become in inequality (43), and hence how close to -1 will $\Gamma(t)$ be driven. From (12) we have

$$
\frac{d}{d t}|\mathbf{r}|=\Gamma(t)|\dot{\mathbf{r}}|,
$$

which from (13) and $|\Gamma(t)| \leq 1, \forall t$, implies

$$
\frac{d}{d t}|\mathbf{r}| \geq-|\Gamma(t)|(1+\nu) \geq-(1+\nu) .
$$

From [45, we conclude that

$$
|\mathbf{r}(t)| \geq|\mathbf{r}(0)|-(1+\nu) t, \quad \forall t \geq 0,
$$

and, more to the point,

$$
|\mathbf{r}(t)| \geq r_{o}, \quad \forall t \leq \frac{|\mathbf{r}(0)|-r_{o}}{1+\nu} .
$$

For (47) to be meaningful for the problem at hand, we assume that $|\mathbf{r}(0)|>r_{o}$. Then defining

$$
T=\frac{|\mathbf{r}(0)|-r_{o}}{1+\nu}>0
$$

to be the minimum interval of time over which we can guarantee that $\dot{\Gamma} \leq 0$, we conclude that

$$
\Gamma(T) \leq \tanh \left(\tanh ^{-1} \Gamma_{0}-c_{2} T\right) .
$$

From [49, we see that by choosing $c_{2}$ sufficiently large (which can be accomplished by choosing $c_{o} \geq 2 c_{1} / \sqrt{\epsilon}$ sufficiently large), we can force $\Gamma(T) \leq-1+\epsilon$. Noting that

$$
\tanh (x) \leq-1+\epsilon \Longleftrightarrow x \leq \frac{1}{2} \ln \left(\frac{\epsilon}{2-\epsilon}\right),
$$

for $0<\epsilon<<1$, we see that

$$
\Gamma(T) \leq-1+\epsilon \Longleftrightarrow \tanh ^{-1} \Gamma_{0}-c_{2} T \leq \frac{1}{2} \ln \left(\frac{\epsilon}{2-\epsilon}\right) .
$$

Thus, if $c_{o} \geq 2 c_{1} / \sqrt{\epsilon}$ is taken to be sufficiently large that

$$
c_{2} \geq(1+\nu) \frac{\tanh ^{-1} \Gamma_{0}-\frac{1}{2} \ln \left(\frac{\epsilon}{2-\epsilon}\right)}{|\mathbf{r}(0)|-r_{o}},
$$

then we are guaranteed (under the conditions mentioned in the above calculations) to achieve $\Gamma\left(t_{1}\right) \leq-1+\epsilon$ at some finite time $t_{1} \leq T$.

\section{B. Statement of result}

Definition: Given the system (1), (2) with $\Gamma$ defined by (12), we say that "motion camouflage is accessible in finite time" if for any $\epsilon>0$ there exists a time $t_{1}>0$ such that $\Gamma\left(t_{1}\right) \leq$ $-1+\epsilon$.

Proposition: Consider the system (1), (2) with $\Gamma$ defined by (12), and control law (30), with the following hypotheses:

(A1) $0<\nu<1$ (and $\nu$ is constant),

(A2) $u_{e}$ is continuous and $\left|u_{e}\right|$ is bounded,

(A3) $\Gamma_{0}=\Gamma(0)<1$, and

(A4) $|\mathbf{r}(0)|>0$.

Motion camouflage is accessible in finite time using high-gain feedback (i.e., by choosing $\mu>0$ sufficiently large).

Proof: Choose $r_{o}>0$ such that $r_{o}<|\mathbf{r}(0)|$. Choose $c_{2}>0$ sufficiently large so as to satisfy (52), and choose $c_{o}$ accordingly to ensure that (36) holds for $\Gamma>-1+\epsilon$. Then defining $\mu$ according to 33 ensures that $\Gamma(T) \leq-1+\epsilon$, where $T>0$ is defined by (48).

Remark: Assumption $(A 1)$ above can be generalized to $0 \leq$ $\nu<1$. (The $\nu=0$ case corresponds to a stationary "evader," so that the natural Frenet frame (2) and steering control $u_{e}$ for the evader are not defined.) 


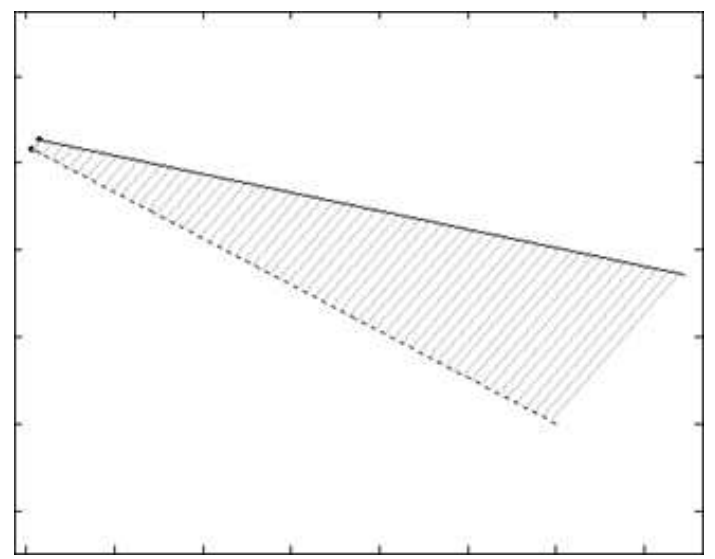

Fig. 2. Straight-line evader trajectory (dashed dark line), and the corresponding pursuer trajectory (solid dark line) evolving according to 11 with control given by 30 .

\section{Simulation RESUlts}

The following simulation results illustrate the behavior of the pursuit-evasion system (1), (2), under the control law (30) for the pursuer and various open-loop controls for the evader. The simulations also confirm the analytical results presented above. Figure 2 shows the behavior of the system for the simplest evader behavior, $u_{e}=0$, which corresponds to straight-line motion. Because control law (30) is the same as (26) when $u_{e}=0, \Gamma$ tends monotonically toward -1 (for the initial conditions and choice of gain $\mu$ used in the simulation shown). In figure 2] as in the subsequent figures showing pursuer and evader trajectories, the solid light lines connect the pursuer and evader positions at evenly-spaced time instants. For a pursuit-evasion system in a state of motion camouflage, these lines would all be parallel to one another. Also, each simulation is run for finite time, at the end of which the pursuer and evader are in close proximity. (The ratio of speeds is $\nu=0.9$ in all of the simulations shown.)

Figure 3 illustrates the behavior of the pursuer for a sinsusoidally-varying steering control $u_{e}$ of the evader, and figure 4 shows the corresponding behavior of $\Gamma(t)$. In figure 4 increasing the value of the feedback gain $\mu$ by a factor of three is observed to decrease the peak difference between $\Gamma$ and -1 by a factor of about $3^{2}=9$. This is consistent with the calculations in the proof of the Proposition. Figure 5 illustrates the behavior of the pursuer for a randomly-varying steering control $u_{e}$ of the evader, and figures 6 and 7 show the corresponding behavior of $\Gamma(t)$. Similarly to figure 4 figure 6 shows that increasing the feedback gain $\mu$ by a factor of three decreases the peak difference between $\Gamma$ and -1 by a factor of about $3^{2}=9$. Figure 7 shows the initial transient in $\Gamma(t)$ for $t$ small. As would be expected, increasing the gain $\mu$ increases the convergence rate. (The time axes for figures 6 and 7 differ by a factor of 200 , which is why the initial transient cannot be seen in figure 6) Finally, figure 8 illustrates the behavior of the pursuer for a constant steering control $u_{e}$, resulting in circling motion by the evader.

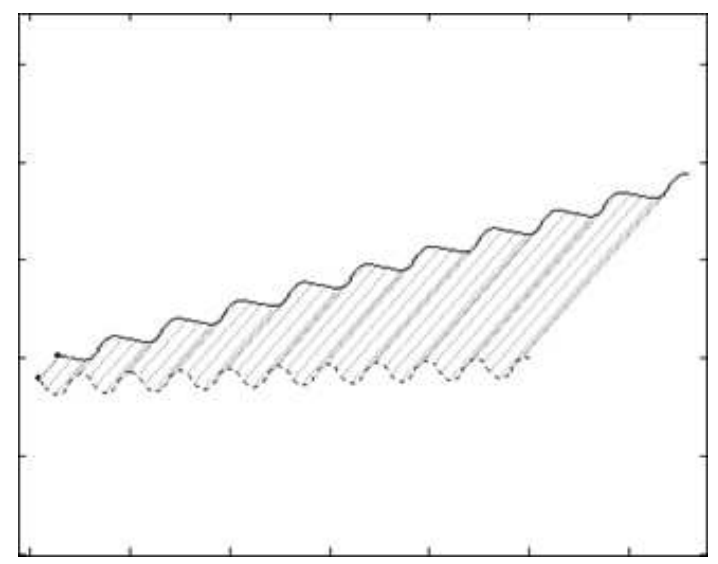

Fig. 3. Evader trajectory with sinusoidally varying steering input (dashed dark line), and the corresponding pursuer trajectory (solid dark line) evolving according to $[1$ with control given by 30 .

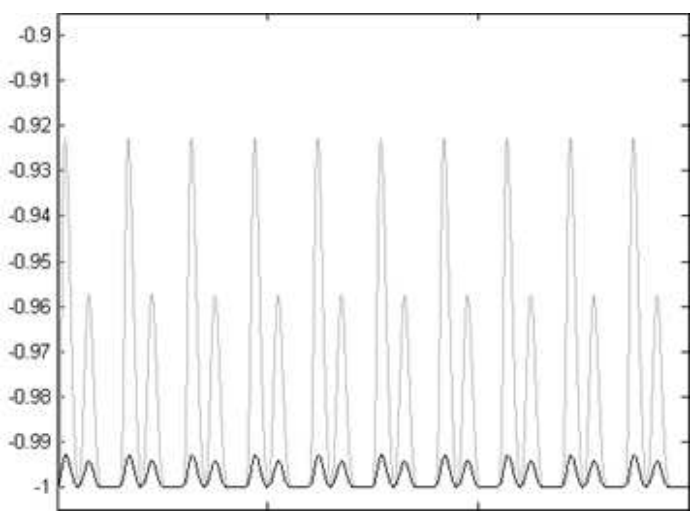

Fig. 4. The cost function $\Gamma(t)$ given by [12], plotted as a function of time, for the pursuit illustrated in figure 3 The two traces correspond to different values of gain $\mu$ : the value of $\mu$ is three times as large for the dark trace as for the light trace. (The trajectories corresponding to the two different gains are qualitatively similar; figure 3 actually corresponds to the lower value of $\mu$.)

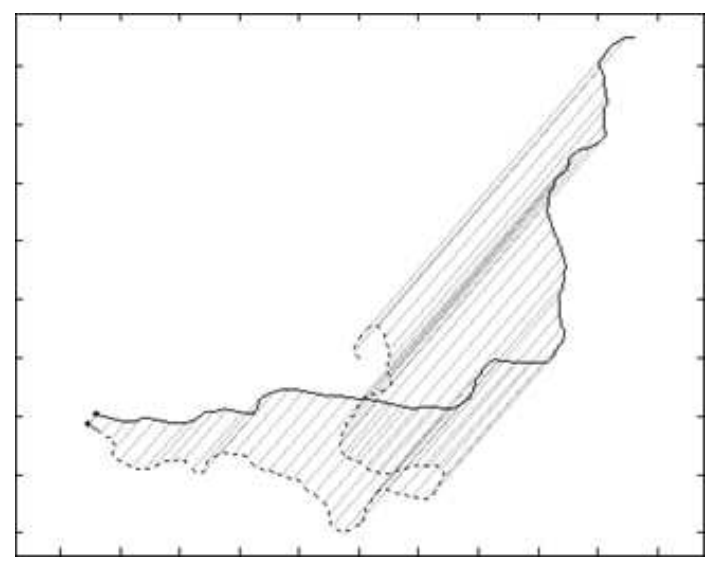

Fig. 5. Evader trajectory with randomly varying steering input (dashed dark line), and the corresponding pursuer trajectory (solid dark line) evolving according to 11 with control given by 30 . 


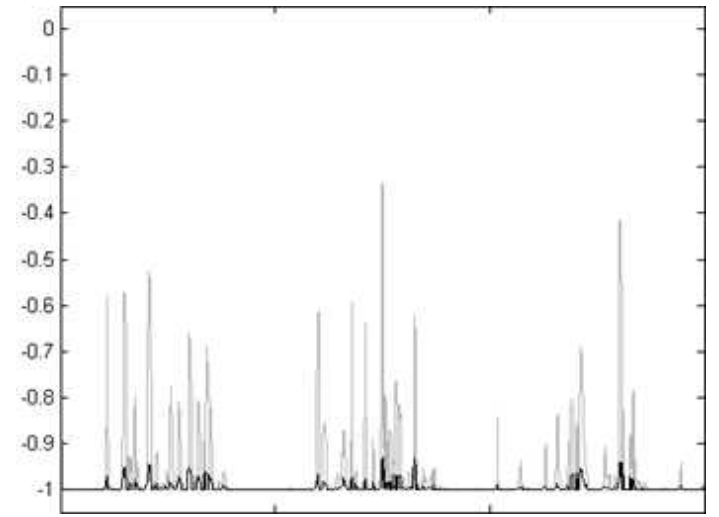

Fig. 6. The cost function $\Gamma(t)$ given by 12 , plotted as a function of time, for the pursuit illustrated in figure 5 The two traces correspond to different values of gain $\mu$ : the value of $\mu$ is three times as large for the dark trace as for the light trace. (The trajectories corresponding to the two different gains are qualitatively similar; figure 5 actually corresponds to the lower value of н.)

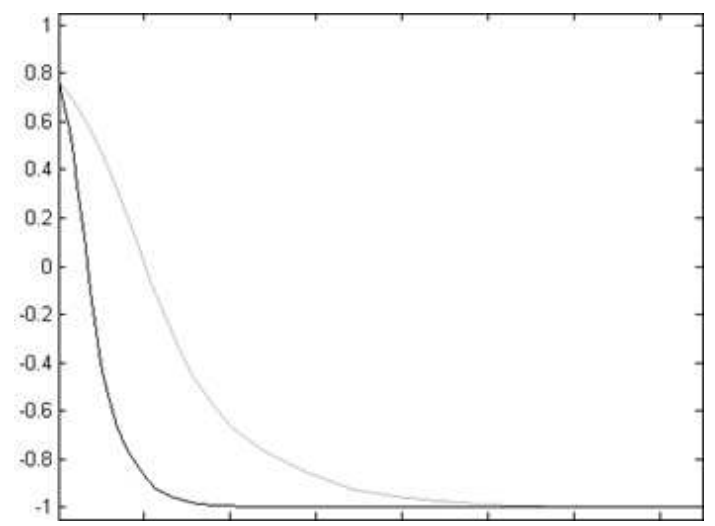

Fig. 7. The cost function $\Gamma(t)$ given by 12, plotted as a function of time, for $1 / 200$ th of the time interval of figure 6 for the pursuit illustrated in figure 5 The two traces correspond to different values of gain $\mu$ : the value of $\mu$ is three times as large for the dark trace as for the light trace. (Because similar initial conditions were used, the expanded-time-scale plot of $\Gamma(t)$ corresponding to figures 3 and 4 is very similar to figure 7 )

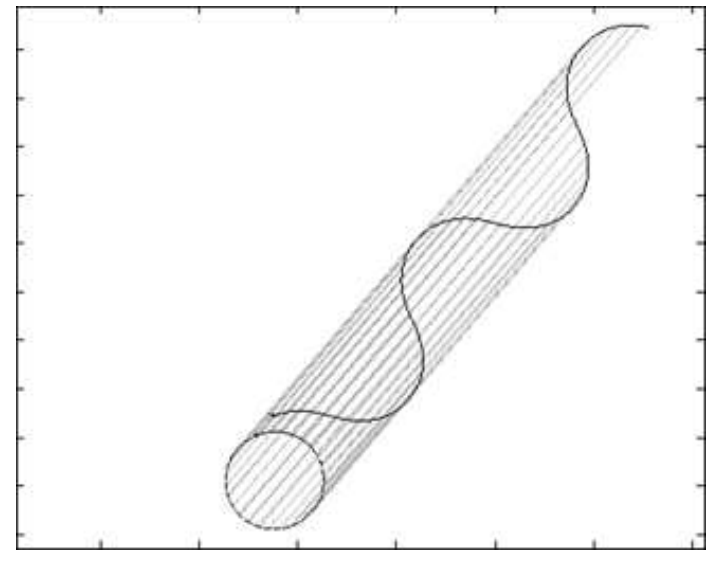

Fig. 8. Evader trajectory with constant steering input (circular trajectory), and the corresponding pursuer trajectory (solid dark line) evolving according to 11 with control given by 30 .

\section{CONNECTIONS TO Missile GUIDANCE}

There is a vast literature on the subject of missile guidance in which the problem of pursuit of an (evasively) maneuvering target by a tactical missile is of central interest. A particular class of feedback laws, known as pure proportional navigation guidance (PPNG) occupies a prominent place [12]. For planar missile-target engagements, the PPNG law determining the steering control for the missile/pursuer is

$$
u^{P P N G}=N \dot{\lambda},
$$

where $\dot{\lambda}$ denotes the rate of rotation (in the plane) of the lineof-sight (LOS) vector from the pursuer to the evader. Here the gain $N$ is a dimensionless positive constant known as the navigation constant. Notice that our motion camouflage guidance law (MCPG) given by (30) has a gain $\mu$ which has the dimensions of $[L E N G T H]^{-1}$. Also, it is easy to see that

$$
\dot{\lambda}=\frac{w}{|\mathbf{r}|}=-\frac{1}{|\mathbf{r}|}\left(\frac{\mathbf{r}}{|\mathbf{r}|} \cdot \dot{\mathbf{r}}^{\perp}\right) .
$$

So, to make a proper comparison we let $r_{o}$ as in Section III be a length scale for the problem and define the dimensionless gain

$$
N^{M C P G}=\mu r_{o} .
$$

Thus, our MPCG law takes the form

$$
u^{M C P G}=N^{M C P G} \frac{|\mathbf{r}|}{r_{o}} \dot{\lambda} .
$$

It follows that motion camouflage uses range information to support a high gain in the initial phase of the engagement, ramping down to a lower value in the terminal phase $\left(|\mathbf{r}| \approx r_{o}\right)$. In nature this extra freedom of gain control is particularly relevant for echolocating bats (see [15]), which have remarkable ranging ability.

Analysis of the performance of the PPNG law is carried out in [13], [14], using arguments similar to ours (although our sufficient conditions appear to be weaker). While motion camouflage as a strategy is discussed in [12], under "parallel navigation," to the best of our knowledge, the current work is the first to present and analyze a feedback law for motion camouflage.

\section{DIRECTIONS FOR FURTHER WORK}

In work under preparation, we have generalized the analysis to the three-dimensional setting, and to planar motion camouflage with respect to a finite point. The three-dimensional analysis is made possible by the use of natural Frenet frames, analogously to the three-dimensional unit-speed particle interaction laws described in [11].

Because we are able to treat the motion camouflage problem within the same framework as our earlier formation control and obstacle-avoidance work [7]-[11], we would like to understand how teams of vehicles can make use of motion camouflage, and whether we can determine the convergence behavior of such systems. Various biologically-inspired scenarios for motion camouflage with teams have been described in [16]. Considering additional military applications without biological analogs, there are thus a variety of team motion camouflage problems to study. 


\section{ACKNOWLEDGEMENTS}

The authors would like to thank M.V. Srinivasan of the Research School of Biological Sciences at the Australian National University for valuable discussions and helpful comments on an earlier draft of this paper.

This research was supported in part by the Naval Research Laboratory under Grants No. N00173-02-1G002, N00173-031G001, N00173-03-1G019, and N00173-04-1G014; by the Air Force Office of Scientific Research under AFOSR Grants No. F49620-01-0415 and FA95500410130; by the Army Research Office under ODDR\&E MURI01 Program Grant No. DAAD19-01-1-0465 to the Center for Communicating Networked Control Systems (through Boston University); and by NIH-NIBIB grant 1 R01 EB004750-01, as part of the NSF/NIH Collaborative Research in Computational Neuroscience Program.

\section{REFERENCES}

[1] M.V. Srinivasan and M. Davey, "Strategies for active camouflage of motion," Proc. Roy. Soc. Lond. B, Vol. 259, No. 1354, pp. 19-25, 1995.

[2] T.S. Collett and M.F. Land, "Visual control of flight behaviour in the hoverfly, Syritta pipiens," J. comp. Physiol., vol. 99, pp. 1-66, 1975.

[3] A.K. Mizutani, J.S. Chahl, and M.V. Srinivasan, "Motion camouflage in dragonflies," Nature, Vol. 423, p. 604, 2003.

[4] M.V. Srinivasan and S. Zhang, "Visual Motor Computations in Insects," Ann. Rev. Neurosci., Vol. 27, pp. 679-696, 2004.

[5] P. Glendinning, "The mathematics of motion camouflage," Proc. Roy. Soc. Lond. B, Vol. 271, No. 1538, pp. 477-481, 2004.

[6] A.J. Anderson and P.W. McOwan, "Model of a predatory stealth behavior camouflaging motion," Proc. Roy. Soc. Lond. B, Vol. 270, No. 1514, pp. 489-495, 2003.

[7] E.W. Justh and P.S. Krishnaprasad, "Equilibria and steering laws for planar formations," Systems and Control Letters, Vol. 51, pp. 25-38, 2004.

[8] E.W. Justh and P.S. Krishnaprasad, "A Simple Control Law for UAV Formation Flying," Institute for Systems Research Technical Report TR 2002-38 (see http://www.isr.umd.edu), 2002.

[9] E.W. Justh and P.S. Krishnaprasad, "Steering laws and continuum models for planar formations," Proc. 42nd IEEE Conf. Decision and Control, pp. 3609-3614, 2003.

[10] F. Zhang, E.W. Justh, and P.S. Krishnaprasad, "Boundary following using gyroscopic control," Proc. 43rd IEEE Conf. Decision and Control, pp. 5204-5209, 2004.

[11] E.W. Justh and P.S. Krishnaprasad, "Natural frames and interacting particles in three dimensions," accepted for 44th IEEE Conf. Decision and Control, December 2005 (arXiv:math.OC/0503390/1).

[12] N.A. Shneydor, Missile Guidance and Pursuit, Horwood, Chichester, 1998.

[13] I.J. Ha, J.H. Hur, M.S. Ko and T.L. Song, "Performance analysis of PNG laws for randomly maneuvering targets," IEEE Trans. Aerospace. Electr. Syst., vol. 26, No. 5, pp. 713-721, 1990.

[14] J.H. Oh and I.J. Ha, "Capturability of the 3-dimensional pure PNG law," IEEE Trans. Aerospace. Electr. Syst., vol. 35, No. 2, pp. 491-503, 1999.

[15] K. Ghose, T. Horiuchi, P.S. Krishnaprasad and C. Moss, "Echolocating bats use a prey intercept strategy that is time-optimal in a local, piecewise linear sense," preprint, June 2005.

[16] A.J. Anderson and P.W. McOwan, "Motion camouflage team tactics," Evolvability \& Interaction Symposium, 2003 (see http://www.dcs.qmul.ac.uk/aaja/TEAM_MC/team_mot_cam.html]. 